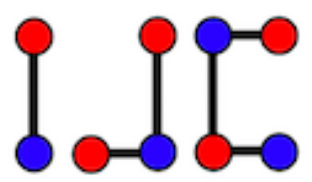

\title{
Triangles in the suborbital graphs of the normalizer of $\Gamma_{0}(N)$
}

\author{
Nazlı Yazıcı Gözütok, Bahadır Özgür Güler \\ Department of Matematics, Karadeniz Technical University, 61080 Trabzon, Turkey \\ nazliyazici@ktu.edu.tr,boguler@ktu.edu.tr
}

\begin{abstract}
In this paper, we investigate a suborbital graph for the normalizer of $\Gamma_{0}(N)$ in $P S L(2, \mathbb{R})$, where $N$ will be of the form $2^{4} p^{2}$, where $p>3$ is a prime number. Then we give edge and circuit conditions on graphs arising from the non-transitive action of the normalizer.

Keywords: congruence subgroups, imprimitive group action, suborbital graphs

Mathematics Subject Classification : O5E18, 11F06, 05C25

DOI: 10.19184/ijc.2020.4.2.1

\section{Introduction}

The modular group acts transitively on $\hat{\mathbb{Q}}$ and in a paper of Jones, Singerman, Wicks, the suborbital graphs were studied and the most basic one turn out to be the well-known Farey graph (See Figure 1) [7]. Suborbital graphs of the normalizer $\operatorname{Nor}(N)$ were studied by same idea. All circuits in the suborbital graph were found when $N$ is a square-free positive integer [9] and when $N$ satisfies the condition of transitive action [10]. Then, non-transitive cases have been examined to reach the general statement $[1,2,5,6,8]$. In this study, we continue to examine the combinatorial properties of the normalizer. This study could be interesting to researchers in the fields algebraic number theory, Diophantine equations and approximations, and algebraic geometry because it is known that related many problems can be described in terms of the Farey graph [11].
\end{abstract}

Received: 11 December 2019, Revised: 17 December 2020, Accepted: 22 December 2020. 


\section{Preliminaries}

$\Gamma_{0}(N)=\left\{\left(\begin{array}{ll}a & b \\ c & d\end{array}\right) \in \Gamma: c \equiv 0 \bmod N\right\}$ is a well known congruence subgroup of the classical modular group $\Gamma$. The normalizer turns to be a very important group in the study of moonshine and for this reason has been studied by many authors [4].

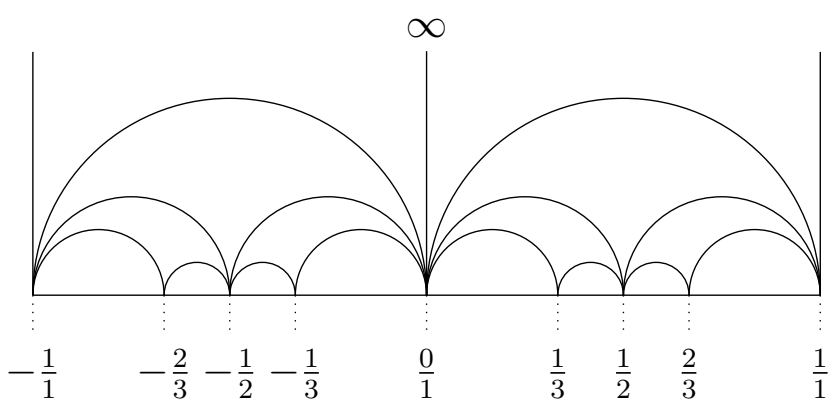

Figure 1. Part of the Farey Graph

The normalizer of $\Gamma_{0}(N)$ consists of the transformations corresponding to the matrices

$$
\left(\begin{array}{cc}
a e & b / h \\
c N / h & d e
\end{array}\right)
$$

where all symbols represent integers, $h$ is the largest divisor of 24 for which $h^{2} \mid N, e>0$ is an exact divisor of $N / h^{2}$ and the determinant is $e$. (We say that $r$ is an exact divisor of $s$ if $r \mid s$ and $(r, s / r)=1)$.

\section{Main Result}

In this study, we take $N=2^{4} p^{2}$, where $p$ is a prime and $p>3$. Then $h=4$ and, hence $e$ must be 1 or $p^{2}$. As a corollary, we get two types of the elements of $\operatorname{Nor}\left(2^{4} p^{2}\right)$ as follows

$$
T_{1}=\left(\begin{array}{cc}
a & b / 4 \\
4 p^{2} c & d
\end{array}\right), a d-b c p^{2}=1, \quad T_{2}=\left(\begin{array}{cc}
a p^{2} & b / 4 \\
4 p^{2} c & d p^{2}
\end{array}\right), a d p^{4}-n b c p^{2}=p^{2} .
$$

Lemma 3.1. Let $N$ be the prime power decomposition as $2^{\alpha_{1}} 3^{\alpha_{2}} p_{1}^{\alpha_{3}} \ldots p_{r}^{\alpha_{r}}$. Then $N$ or $(N)$ acts transitively on $\hat{\mathbb{Q}}$ if and only if $\alpha_{1} \leq 7, \alpha_{2} \leq 3$ and $\alpha_{i} \leq 1$ for $i=3, \ldots, r$.

Corollary 3.1. $\operatorname{Nor}\left(2^{4} p^{2}\right)$ is not transitive on $\hat{\mathbb{Q}}$.

Therefore, we will find a maximal subset of $\hat{\mathbb{Q}}$ on which $\operatorname{Nor}\left(2^{4} p^{2}\right)$ acts transitively. 
Triangles in the suborbital graphs of the normalizer of $\Gamma_{0}(N) \quad \mid \quad$ N.Y. Gözütok and B.Ö. Güler

Lemma 3.2. Let $d \mid N$. Then the orbit $\left(\begin{array}{l}a \\ d\end{array}\right)$ of $\frac{a}{d}$ with $(a, d)=1$ under $\Gamma_{0}(N)$ is the set

$$
\left\{\frac{x}{y} \in \hat{\mathbb{Q}}:(N, y)=d, a \equiv x \frac{y}{d} \quad \bmod \left(d, \frac{N}{d}\right)\right\}
$$

with $d \mid N$ under $\Gamma_{0}(N)$ is just $\varphi\left(d, \frac{N}{d}\right)$, where $\varphi$ is the Euler function.

Hence, we can give following tables by above lemma:

Table 1. Divisors of $N / h^{2}$

\begin{tabular}{lllll}
\hline 1 & 2 & $2^{2}$ & $2^{3}$ & $2^{4}$ \\
\hline$p$ & $2 p$ & $2^{2} p$ & $2^{3} p$ & $2^{4} p$ \\
\hline$p^{2}$ & $2 p^{2}$ & $2^{2} p^{2}$ & $2^{3} p^{2}$ & $2^{4} p^{2}$ \\
\hline
\end{tabular}

Table 2. Number of Orbits

\begin{tabular}{lllll}
\hline 1 & 1 & 2 & 1 & 1 \\
\hline$p-1$ & $p-1$ & $2 p-2$ & $p-1$ & $p-1$ \\
\hline 1 & 1 & 2 & 1 & 1 \\
\hline
\end{tabular}

Theorem 3.1. The maximal subset of $\hat{\mathbb{Q}}$ on which $N$ or $\left(2^{4} p^{2}\right)$ acts transitively is

$$
\begin{aligned}
& \hat{\mathbb{Q}}\left(2^{4} p^{2}\right)=\left(\begin{array}{l}
1 \\
1
\end{array}\right) \cup\left(\begin{array}{l}
1 \\
2
\end{array}\right) \cup\left(\begin{array}{c}
1 \\
2^{2}
\end{array}\right) \cup\left(\begin{array}{c}
1 \\
2^{3}
\end{array}\right) \cup\left(\begin{array}{c}
1 \\
2^{4}
\end{array}\right) \cup\left(\begin{array}{c}
1 \\
p^{2}
\end{array}\right) \cup\left(\begin{array}{c}
1 \\
2 p^{2}
\end{array}\right) \cup\left(\begin{array}{c}
1 \\
2^{2} p^{2}
\end{array}\right) \\
& \cup\left(\begin{array}{c}
1 \\
2^{3} p^{2}
\end{array}\right) \cup\left(\begin{array}{c}
1 \\
2^{4} p^{2}
\end{array}\right),
\end{aligned}
$$

where $\left(\begin{array}{l}x \\ y\end{array}\right)$ denotes the $\operatorname{Nor}\left(2^{4} p^{2}\right)$-orbit of $\frac{x}{y} \in \hat{\mathbb{Q}}$.

Proof. It is sufficient to find the images of the orbit $\left(\begin{array}{l}1 \\ 1\end{array}\right)$ under the transformations of $\operatorname{Nor}\left(2^{4} p^{2}\right)$. Since there are two different types of transformations in the normalizer $\operatorname{Nor}\left(2^{4} p^{2}\right)$, it is necessary to examine the action of the transformations one by one. For $T_{1}=\left(\begin{array}{cc}a & b / 4 \\ 4 p^{2} c & d\end{array}\right), a d-b c p^{2}=1$, it is obtained

$$
\left(\begin{array}{cc}
a & b / 4 \\
4 p^{2} c & d
\end{array}\right)\left(\begin{array}{l}
1 \\
1
\end{array}\right)=\left(\begin{array}{c}
4 a+b \\
4\left(4 p^{2} c+d\right)
\end{array}\right) .
$$

Then, we have 
Triangles in the suborbital graphs of the normalizer of $\Gamma_{0}(N) \quad \mid \quad$ N.Y. Gözütok and B.Ö. Güler

i) If $b$ and $d$ are odd, then $\frac{4 a+b}{4\left(4 p^{2} c+d\right)} \in\left(\begin{array}{c}1 \\ 2^{2}\end{array}\right)$, i.e. $T_{1}\left(\begin{array}{c}1 \\ 1\end{array}\right) \in\left(\begin{array}{c}1 \\ 2^{2}\end{array}\right)$.

ii) Let $b$ be odd and $d$ be even.
1) If $2 \| d$, then $T_{1}\left(\begin{array}{l}1 \\ 1\end{array}\right) \in\left(\begin{array}{c}1 \\ 2^{3}\end{array}\right)$.
2) If $2^{2} \| d$, then $T_{1}\left(\begin{array}{l}1 \\ 1\end{array}\right) \in\left(\begin{array}{c}1 \\ 2^{4}\end{array}\right)$.

iii) Let $b$ be even and $d$ be odd.

1) If $2 \| b$, then $T_{1}\left(\begin{array}{l}1 \\ 2\end{array}\right) \in\left(\begin{array}{c}1 \\ 2^{3}\end{array}\right)$.

2) If $2^{2} \| b$, then $T_{1}\left(\begin{array}{l}1 \\ 1\end{array}\right) \in\left(\begin{array}{l}1 \\ 1\end{array}\right)$.

For $T_{2}=\left(\begin{array}{cc}a p^{2} & b / 4 \\ 4 p^{2} c & d p^{2}\end{array}\right), a d p^{2}-b c=1$, it is obtained

$$
\left(\begin{array}{cc}
a p^{2} & b / 4 \\
4 p^{2} c & d p^{2}
\end{array}\right)\left(\begin{array}{l}
1 \\
1
\end{array}\right)=\left(\begin{array}{c}
4 a p^{2}+b \\
4 p^{2}(4 c+d)
\end{array}\right)
$$

Therefore, we have

i) If $b$ and $d$ are odd, then $T_{2}\left(\begin{array}{l}1 \\ 1\end{array}\right) \in\left(\begin{array}{c}1 \\ 2^{2} p^{2}\end{array}\right)$.

ii) Let $b$ be odd and $d$ be even.

1) If $2 \| d$, then $T_{2}\left(\begin{array}{l}1 \\ 1\end{array}\right) \in\left(\begin{array}{c}1 \\ 2^{3} p^{2}\end{array}\right)$.

2) If $2^{2} \| d$, then $T_{2}\left(\begin{array}{l}1 \\ 1\end{array}\right) \in\left(\begin{array}{c}1 \\ 2^{4} p^{2}\end{array}\right)$.

iii) Let $b$ be even and $d$ be odd.

1) If $2 \| b$, then $T_{1}\left(\begin{array}{l}1 \\ 1\end{array}\right) \in\left(\begin{array}{c}1 \\ 2 p^{2}\end{array}\right)$.

2) If $2^{2} \| b$, then $T_{1}\left(\begin{array}{l}1 \\ 1\end{array}\right) \in\left(\begin{array}{c}1 \\ p^{2}\end{array}\right)$. 
Lemma 3.3. [3] Let $(G, \Delta)$ be a transitive permutation group. $(G, \Delta)$ is primitive if and only if $S_{\alpha}$, the stabilizer of $\alpha \in \Delta$, is a maximal subgroup of $G$ for each $\alpha \in \Delta$.

From the above lemma we see that whenever, for some $\alpha, S_{\alpha} \lesseqgtr H \lesseqgtr G$, then $\Delta$ admits some $G$-invariant equivalence relation other than the trivial cases. Because of the transitivity, every element of $\Delta$ has the form $g(\alpha)$ for some $g \in G$. Thus, one of the non-trivial $G$-invariant equivalence relations on $\Delta$ is given as follows:

$$
g(\alpha) \approx g^{\prime}(\alpha) \text { if and only if } g^{\prime} \in g H .
$$

The number of blocks is the index $|G: H|$ and the block containing $\alpha$ is just the orbit $H(\alpha)$.

To apply these ideas to the case where $G$ is $\operatorname{Nor}\left(2^{4} p^{2}\right)$, and $\Delta$ is $\hat{\mathbb{Q}}\left(2^{4} p^{2}\right)$. Also we find the stabilizer of $\infty$ in $\operatorname{Nor}\left(2^{4} p^{2}\right)$, say $\operatorname{Nor}\left(2^{4} p^{2}\right)_{\infty}$, as the cyclic group generated by $\left(\begin{array}{cc}1 & 1 / 4 \\ 0 & 1\end{array}\right)$. Thus we take

$$
\operatorname{Nor}\left(2^{4} p^{2}\right)_{\infty}=\left\langle\left(\begin{array}{cc}
1 & 1 / 4 \\
0 & 1
\end{array}\right)\right\rangle
$$

and $\Gamma_{C}(N)$ instead of $H$ and $S_{\alpha}$, respectively. Clearly $N$ or $\left.\left(2^{4} p^{2}\right)_{\infty} \lesseqgtr \Gamma_{C}(N)\right) \lesseqgtr N o r\left(2^{4} p^{2}\right)$.

Now, we give the following lemma which helps in calculating the number of blocks.

Theorem 3.2. The blocks arising from the imprimitive action are

$$
\begin{aligned}
& {[0]=\left(\begin{array}{l}
1 \\
1
\end{array}\right) \cup\left(\begin{array}{l}
1 \\
2
\end{array}\right) \cup\left(\begin{array}{c}
1 \\
2^{2}
\end{array}\right) \cup\left(\begin{array}{c}
1 \\
2^{3}
\end{array}\right) \cup\left(\begin{array}{c}
1 \\
2^{4}
\end{array}\right)} \\
& {[\infty]=\left(\begin{array}{c}
1 \\
p^{2}
\end{array}\right) \cup\left(\begin{array}{c}
1 \\
2 p^{2}
\end{array}\right) \cup\left(\begin{array}{c}
1 \\
2^{2} p^{2}
\end{array}\right) \cup\left(\begin{array}{c}
1 \\
2^{3} p^{2}
\end{array}\right) \cup\left(\begin{array}{c}
1 \\
2^{4} p^{2}
\end{array}\right) .}
\end{aligned}
$$

Proof. Since $N=2^{4} p^{2}, h=2^{2}$ and $N / h^{2}=p^{2}$. So the number of distinct prime factors of $p^{2}$ is $\rho=1$. Then, $\operatorname{Nor}\left(2^{4} p^{2}\right): \Gamma_{C}\left(2^{4} p^{2}\right) \mid=2$. The result is obvious.

Let $\Delta$ be a set and $G$ be a permutation group acting on $\Delta$. The graphs arising from this action is called the suborbital graphs of $G$ [12]. Also, the set $\Delta$ is the set of vertices of these graphs. More clearly, the action of $G$ on $\Delta \times \Delta$ given by $g(\alpha, \beta)=(g(\alpha), g(\beta)),(g \in G, \alpha, \beta \in \Delta)$. Using this action, one can obtain orbits called suborbitals of $G$. Let the orbit of the element $(\alpha, \beta) \in \Delta \times \Delta$ be $O(\alpha, \beta)$. Using $O(\alpha, \beta)$ we can construct a suborbital graph $G(\alpha, \beta)$ as follows: the set of vertices is the set $\Delta$, and if $(\gamma, \delta) \in O(\alpha, \beta)$, then there exists an directed edge from $\gamma$ to $\delta$. This directed edge is represented by $(\gamma \rightarrow \delta)$. If there exists a directed edge from $\gamma$ to $\delta$, it is said that the corresponding suborbital graph contains an edge from $\gamma$ to $\delta$. Let $v_{1} \rightarrow v_{2} \rightarrow \cdots \rightarrow v_{m} \rightarrow v_{1}$ be a sequence of edges, where $v_{i} \neq v_{j}$ for $i \neq j$ and $m \geq 3$, this sequence is called a circuit of length $m$. In particular, the circuit is called a triangle or a quadrilateral if its length is 3 or 4 , respectively.

In this study, $G$ is $N o r\left(2^{4} p^{2}\right)$ and $\Delta$ is $\hat{\mathbb{Q}}\left(2^{4} p^{2}\right)$. We now consider the suborbital graphs for the action of $\operatorname{Nor}\left(2^{4} p^{2}\right)$ on $\hat{\mathbb{Q}}\left(2^{4} p^{2}\right)$. Since $\operatorname{Nor}\left(2^{4} p^{2}\right)$ acts transitively on $\hat{\mathbb{Q}}\left(2^{4} p^{2}\right), \operatorname{Nor}\left(2^{4} p^{2}\right)$ 
permutes the blocks transitively, so the subgraphs are all isomorphic. Thus it is sufficient to study with only one block. On the other hand, it is clear that each non-trivial suborbital graph contains a pair $\left(\infty, \frac{u}{p^{2}}\right)$ for some $\frac{u}{p^{2}} \in \hat{\mathbb{Q}}\left(2^{4} p^{2}\right)$, where $\left(u, p^{2}\right)=1$. We denote by $F_{u, p^{2}}$ the subgraph of $G\left(\infty, \frac{u}{p^{2}}\right)$ whose vertices are in the block

$$
[\infty]=\left(\begin{array}{c}
1 \\
p^{2}
\end{array}\right) \cup\left(\begin{array}{c}
1 \\
2 p^{2}
\end{array}\right) \cup\left(\begin{array}{c}
1 \\
2^{2} p^{2}
\end{array}\right) \cup\left(\begin{array}{c}
1 \\
2^{3} p^{2}
\end{array}\right) \cup\left(\begin{array}{c}
1 \\
2^{4} p^{2}
\end{array}\right)
$$

so that $G\left(\infty, \frac{u}{p^{2}}\right)$ consists of two disjoint copies $F_{u, p^{2}}$.

Theorem 3.3. Let $\frac{r}{s}, \frac{x}{y} \in[\infty]$. Then $\frac{r}{s} \rightarrow \frac{x}{y}$ is an edge in $F_{u, p^{2}}$ if and only if

(i) If $16 p^{2} \| s$, then $x \equiv \pm u r\left(\bmod p^{2}\right), y \equiv \pm u s\left(\bmod p^{2}\right), r y-s x= \pm p^{2}$,

(ii) If $4 p^{2} \| s$, then $x \equiv \pm 4 u r\left(\bmod p^{2}\right), y \equiv \pm 4 u s\left(\bmod p^{2}\right), r y-s x= \pm 4 p^{2}$,

(iii) If $p^{2} \| s$, then $x \equiv \pm 16 u r\left(\bmod p^{2}\right), y \equiv \pm 16 u s\left(\bmod p^{2}\right), r y-s x= \pm p^{2}$.

Proof. Let $\frac{r}{s} \gg \frac{x}{y}$ be an edge in $F_{u, p^{2}}$. Then there exists an element $T \in \operatorname{Nor}\left(2^{4} p^{2}\right)$ such that $T(\infty)=\frac{r}{s}$ and $T\left(\frac{u}{p^{2}}\right)=\frac{x}{y}$. Now, suppose $16 p^{2} \| s$. Thus, $T$ is of the form $\left(\begin{array}{cc}a & b \\ 16 p^{2} c & d\end{array}\right)$ with $a d-16 b c p^{2}=1$. Thus, $\left(\begin{array}{cc}a & b \\ 16 p^{2} c & d\end{array}\right)\left(\frac{1}{0}\right)=\frac{a}{16 p^{2} c}=\frac{r}{s}$. By determinant, $a$ is odd, so we have $r=a$ and $s=16 p^{2} c$. Also

$$
\left(\begin{array}{cc}
a & b \\
16 p^{2} c & d
\end{array}\right)\left(\frac{u}{p^{2}}\right)=\frac{a u+b p^{2}}{16 p^{2} c u+d p^{2}}=\frac{x}{y},
$$

then $x=a u+b p^{2}$ and $y=16 p^{2} c u+d p^{2}$. Using $r=a$ and $s=16 p^{2} c$, it is obtained $x \equiv \operatorname{ur}\left(\bmod p^{2}\right)$ and $y \equiv u s\left(\bmod p^{2}\right)$. Using determinants in the following equation

$$
\left(\begin{array}{cc}
a & b \\
16 p^{2} c & d
\end{array}\right)\left(\begin{array}{cc}
1 & u \\
0 & p^{2}
\end{array}\right)=\left(\begin{array}{cc}
a & a u+b p^{2} \\
16 p^{2} c & 16 p^{2} c u+d p^{2}
\end{array}\right)=\left(\begin{array}{ll}
r & x \\
s & y
\end{array}\right)
$$

we have $r y-s x=p^{2}$.

Suppose $4 p^{2} \| s$. Thus, $T$ is of the form $\left(\begin{array}{cc}a & b / 4 \\ 4 p^{2} c & d\end{array}\right)$ with $a d-b c p^{2}=1$. Thus,

$$
\left(\begin{array}{cc}
a & b / 4 \\
4 p^{2} c & d
\end{array}\right)\left(\frac{1}{0}\right)=\frac{a}{16 p^{2} c}=\frac{r}{s} .
$$

By determinant, $a$ is odd, so we have $r=a$ and $s=4 p^{2} c$. Also

$$
\left(\begin{array}{cc}
a & b / 4 \\
4 p^{2} c & d
\end{array}\right)\left(\frac{u}{p^{2}}\right)=\frac{4 a u+b p^{2}}{16 p^{2} c u+4 d p^{2}}=\frac{x}{y},
$$


then $x=4 a u+b p^{2}$ and $y=16 p^{2} c u+4 d p^{2}$. Using $r=a$ and $s=4 p^{2} c$, it is obtained $x \equiv$ $4 u r\left(\bmod p^{2}\right)$ and $y \equiv 4 u s\left(\bmod p^{2}\right)$. Using determinants in the following equation

$$
\left(\begin{array}{cc}
4 a & b \\
4 p^{2} c & d
\end{array}\right)\left(\begin{array}{cc}
1 & u \\
0 & p^{2}
\end{array}\right)=\left(\begin{array}{cc}
4 a & 4 a u+b p^{2} \\
4 p^{2} c & 4 p^{2} c u+d p^{2}
\end{array}\right)=\left(\begin{array}{cc}
4 r & x \\
s & y / 4
\end{array}\right),
$$

we have $r y-s x=4 p^{2}$.

Suppose $p^{2} \| s$. Thus, $T$ is of the form $\left(\begin{array}{cc}a & b / 4 \\ 4 p^{2} c & d\end{array}\right)$ with $a d-b c p^{2}=1$. Thus,

$$
\left(\begin{array}{cc}
a & b / 4 \\
4 p^{2} c & d
\end{array}\right)\left(\frac{1}{0}\right)=\frac{a}{4 p^{2} c}=\frac{r}{s} .
$$

Let $a$ be odd, so we have $r=a$ and $s=p^{2} c$. Also

$$
\left(\begin{array}{cc}
a & b / 4 \\
4 p^{2} c & d
\end{array}\right)\left(\frac{u}{p^{2}}\right)=\frac{4 a u+b p^{2}}{16 p^{2} c u+4 d p^{2}}=\frac{x}{y},
$$

then $x=4 a u+b p^{2}$ and $y=16 p^{2} c u+4 d p^{2}$. Using $r=a$ and $s=p^{2} c$, it is obtained $x \equiv$ $16 u r\left(\bmod p^{2}\right)$ and $y \equiv 16 u s\left(\bmod p^{2}\right)$. Using determinants in the following equation

$$
\left(\begin{array}{cc}
4 a & b \\
4 p^{2} c & d
\end{array}\right)\left(\begin{array}{cc}
1 & u \\
0 & p^{2}
\end{array}\right)=\left(\begin{array}{cc}
4 a & 4 a u+b p^{2} \\
4 p^{2} c & 4 p^{2} c u+d p^{2}
\end{array}\right)=\left(\begin{array}{cc}
16 r & x \\
4 s & y / 4
\end{array}\right)
$$

we have $r y-s x=p_{r}^{2}$.

In a similar way, $\frac{r}{s} \rightarrow \frac{x}{y}$ could be shown.

For the opposite, we show only $(i i i)$ for plus sign, the others could be shown similarly. Assume $p^{2} \| s, x \equiv 16 u r\left(\bmod p^{2}\right), y \equiv \pm 16 u s\left(\bmod p^{2}\right), r y-s x=p^{2}$. There exist $m, n \in \mathbb{Z}$ such that $x=16 u r+m p^{2}$ and $y=16 u s+n p^{2}$. Using $r y-s x=p^{2}$, we get $r n-s m=1$. Consider the transformation $T=\left(\begin{array}{cc}r & m \\ s & n\end{array}\right)$. This matrix has determinant 1. It is easily seen that $T(\infty)=\frac{r}{s}$ and $T\left(\frac{u}{p^{2}}\right)=\frac{x}{y}$. So, $\frac{r}{s} \rightarrow \frac{x}{y}$ is an edge in $F_{u, p^{2}}$.

Theorem 3.4. $F_{u, p^{2}}$ contains a triangle if and only if $16 u^{2} \pm 4 u+1 \equiv 0\left(\bmod p^{2}\right)$.

Proof. Assume that there exists a triangle in $F_{u, n}$. By the transitivity of the action, it has the form

$$
\frac{1}{0} \rightarrow \frac{u}{p^{2}} \rightarrow \frac{x}{y} \rightarrow \frac{1}{0}
$$

For the edge $\frac{x}{y} \rightarrow \frac{1}{0}$, then we have $y=p^{2}$ or $y=4 p^{2}$. Let $y=p^{2}$. The triangle takes the form of

$$
\frac{1}{0} \rightarrow \frac{u}{p^{2}} \lesseqgtr \frac{x}{p^{2}} \rightarrow \frac{1}{0} .
$$


For the edge $\frac{u}{p^{2}} \rightarrow \frac{x}{p^{2}}$, by Theorem 3.3 , then we have $x \equiv-16 u^{2}\left(\bmod p^{2}\right)$ and $x=u+1$.

For the edge $\frac{x}{p^{2}} \rightarrow \frac{1}{0}$, by the Theorem 3.3 , then we have, $1 \equiv-16 u x\left(\bmod p^{2}\right)$ and $x=u+1$. Using these equations we conclude $16 u^{2}+16 u+1 \equiv 0\left(\bmod p^{2}\right)$. These equivalences gives a contradiction. So, $y=4 p^{2}$ and the triangle has the form

$$
\frac{1}{0} \rightarrow \frac{u}{p^{2}} \rightarrow \frac{x}{4 p^{2}} \rightarrow \frac{1}{0}
$$

For the edge $\frac{u}{p^{2}} \stackrel{<}{\rightarrow} \frac{x}{4 p^{2}}$, by Theorem 3.3 , then we have $x \equiv-16 u^{2}\left(\bmod p^{2}\right)$ and $x=4 u+1$.

For the edge $\frac{x}{4 p^{2}} \lesseqgtr \frac{1}{0}$, by the Theorem 3.3 , then we have, $1 \equiv-4 u x\left(\bmod p^{2}\right)$ and $x=4 u+1$. Using these equations we conclude $16 u^{2}+4 u+1 \equiv 0\left(\bmod p^{2}\right)$. Conversely, let $16 u^{2} \pm 4 u+1 \equiv$ $0\left(\bmod p^{2}\right)$. Consider the triangle

$$
\frac{1}{0} \rightarrow \frac{u}{p^{2}} \rightarrow \frac{4 u \pm 1}{4 p^{2}} \rightarrow \frac{1}{0}
$$

It is easily shown that each edge of this triangle is in $F_{u, p^{2}}$, by using the conditions Theorem 3.3 respectively.

Example 1. As an simplest example, suppose that $p$ is equal to 7 . We calculate which suborbital graphs contains a triangle. Since $16 u^{2}+4 u+1 \equiv 0\left(\bmod 7^{2}\right)$, then $16 u^{2}+4 u+1 \equiv 0(\bmod 7)$, giving $u=4+7 k$ such that $k \in \mathbb{Z}$. Hence, we have $16(4+7 k)^{2}+4(4+7 k)+1 \equiv 0\left(\bmod 7^{2}\right)$, then $784 k^{2}+924 k+273 \equiv 0\left(\bmod 7^{2}\right)$. As $112 k^{2}+132 k+39 \equiv 0(\bmod 7)$, we obtain $k=4$ and $u=32$. Since $16(32)^{2}+4(32)+1 \equiv 0(\bmod 49), F(\infty, 32 / 49)$ contains a triangle.

Theorem 3.5. The prime divisors $p$ of $16 u^{2} \pm 4 u+1$, for any $u \in \mathbb{Z}$, are of the form $p \equiv 1$ $(\bmod 3)$.

Proof. Let $u$ be any integer and $p$ a prime divisor of $16 u^{2} \pm 4 u+1$. Then, without any difficulty, it can be easily seen that the normalizer $\operatorname{Nor}\left(2^{4} p\right)$, like $\operatorname{Nor}\left(2^{4} p^{2}\right)$, has the elliptic element

$$
\varphi:=\left(\begin{array}{cc}
-2^{4} u & \left(16 u^{2} \pm 4 u+1\right) / p \\
-2^{4} p & 2^{4} u+4
\end{array}\right)
$$

of order 3. From [1, Theorem 2], we get that $p \equiv 1(\bmod 3)$.

\section{Conclusion}

It may be a good idea to examine the graphs corresponding to a group to understand the structure of that group. In this context, it is important to understand the suborbital graphs of the normalizer of $\Gamma_{0}(N)$ in $P S L(2, \mathbb{R})$ in order to reveal at least the combinatorial properties of the normalizer whose importance has emerged after Conway and Norton's famous study [4]. Therefore, in the present study we investigate the suborbital graphs of the normalizer for a non-transitive action. First we define an imprimitive action, and then introduce the suborbital graphs $F_{u, p^{2}}$ of the normalizer. These suborbital graphs does not have to contain a circuit for all pairs $\left(u, p^{2}\right)$. As our main result, we show that $F_{u, p^{2}}$ contains a triangle if and only if $16 u^{2} \pm 4 u+1 \equiv 0\left(\bmod p^{2}\right)$. 
Triangles in the suborbital graphs of the normalizer of $\Gamma_{0}(N) \quad \mid \quad$ N.Y. Gözütok and B.Ö. Güler

\section{References}

[1] M. Akbas and D. Singerman, The signature of the normalizer of $\Gamma_{0}(N)$, Lond. Math. Soc. Lect. Note Ser. 165 (1992), 77-86.

[2] M. Beşenk, B.Ö. Güler, and A. Buyukkaya, Suborbital graphs for a non-transitive action of the normalizer, Filomat 33 (2019), 385-392.

[3] N.L. Biggs and A.T. White, Permutation Groups and Combinatorial Structures, Cambridge University Press, Cambridge, 1979.

[4] J.H. Conway and S.P. Norton, Monstrous moonshine, Bull. Lond. Math. Soc. 11 (1977), 308339.

[5] B.Ö. Güler, M. Beşenk and S. Kader, On congruence equations arising from suborbital graphs, Turk. J. Math. 43(5) (2019), 2369-2404.

[6] B.Ö. Güler, M. Beşenk, A.H. Değer and S. Kader, Elliptic elements and circuits in suborbital graphs, Hacet. J. Math. Stat. 40(2) (2011), 203-210.

[7] G.A. Jones, D. Singerman and K. Wicks, The modular group and generalized Farey graphs, Lond. Math. Soc. Lect. Note Ser. 160 (1991), 316-338.

[8] S. Kader, Circuits in suborbital graphs for the normalizer, Graphs Combin. 33 (2017), 15311542.

[9] R. Keskin, Suborbital graphs for the normalizer $\Gamma_{0}(m)$, Eur. J. Combin. 27 (2006), 193-206.

[10] R. Keskin and B. Demirtürk, On suborbital graphs for the normalizer of $\Gamma_{0}(m)$, Electron. $J$. Combin. 16 (2009), 1-18.

[11] I. Niven, H.S. Zuckerman and H.L. Montgomery, An Introduction to the Theory of Numbers, John Wiley Sons Inc, New York, 1991.

[12] C.C. Sims, Graphs and finite permutation groups, Math. Z. 95 (1967), 76-86. 